\title{
DETERMINACIÓN DE LA PRESENCIA DE TUBERCULOSIS BOVINA EN LA PROVINCIA DE CANTA, LIMA
}

\author{
Flor Flores C. ${ }^{1}$, Alfredo Delgado C. ${ }^{2}$, Armando González Z. ${ }^{3}$ y Hermelinda Rivera G. ${ }^{4}$
}

\section{Abstract}

The presence of bovine tuberculosis was determined in 503 cattle older than four weeks from Canta province, Lima by the tuberculin skin test (PPD). Lecture of the test was performed 72 hours after intradermal injection. Eleven out of 503 animals (2.2\%) were positive to cutaneous tuberculin stimuli. These results were analyzed by the risk evaluation technique using Monte Carlo simulation (@ Risk Program) and the probability of finding the infection in cattle from Canta province resulted greater than $1 \%$ and less than $3.2 \%$.

Key words: bovine tuberculosis, tuberculin, PPD, probability

\section{Resumen}

La presencia de tuberculosis bovina fue determinada en 503 bovinos mayores de 4 semanas de edad en la provincia de Canta, departamento de Lima, con la prueba de tuberculina (PPD). La lectura de la prueba fue realizada 72 horas después de la inyección intradérmica. Once de 503 bovinos $(2.2 \%)$ fueron reactores positivos a la prueba de tuberculina. Estos resultados fueron analizados por la técnica de evaluación de riesgos por simulación Monte Carlo (programa @ Risk) indicando que la probabilidad de encontrar un animal infectado en los bovinos de la provincia de Canta; Lima, es mayor al $1 \%$ y menor al $3.2 \%$.

Palabras clave: tuberculosis bovina, tuberculina, PPD, probabilidad

\footnotetext{
${ }^{1}$ Práctica privada

${ }^{2}$ Clínica de Animales Mayores, FMV-UNMSM.E-mail: aldelgado@viabcp.com

${ }^{3}$ Laboratorio de Medicina Veterinaria Preventiva, FMV-UNMSM

${ }^{4}$ Laboratorio de Microbiología y Parasitología Veterinaria, FMV-UNMSM
} 


\section{INTRODUCCIÓN}

La tuberculosis es una de las enfermedades infecciosas que afecta al ser humano de mayor mortalidad a nivel mundial (Langeneger y Oliveira, 1981). La infección con Mycobacterium bovis (M. bovis) es responsable de aproximadamente 7,000 casos nuevos de tuberculosis humana por año en América Latina (Toledo et al., 1999). La facilidad y frecuencia con que la tuberculosis de los animales se extiende a las personas en un medio no controlado convierten a esta enfermedad en una zoonosis importante (O’Reilley y Daborn, 1995; Radostits et al., 2002).

La tuberculosis bovina se concentra principalmente en países en desarrollo y en ganaderías de crianza intensiva (Sommerfelt, 1989). La prevalencia en Europa es menor de $0.1 \%$ (Acha y Szyfres, 2003), en tanto que en Sudamérica se encuentra alrededor del 1\% (OPS/OMS, 1992). En el país, Castagnino (1968) reportó el 37.6 y 18.1\% en Lima y a nivel nacional, respectivamente; pero estudios actuales realizados por el Servicio Nacional de Sanidad Agraria (SENASA) indican prevalencias inferiores al $1 \%$ en establos lecheros bajo control oficial.

El M. bovis es el agente causal específico de la tuberculosis del ganado vacuno (Acha y Szyfres, 2003; Radostits et al., 2002); sin embargo, todas las especies, incluidos los seres humanos, son susceptibles al M. bovis (Beer, 1981; Radostits et al., 2002).

Las micobacterias cuentan como mínimo con 50 determinantes antigénicos diferentes. Entre las diversas especies y las corinebacterias, nocardias y rhodococos existen antígenos cruzados, entre ellos, los de mayor importancia se tiene el $M$. avium, $M$. intracellulare y dependiendo de la exposición humana, también el $M$. tuberculosis (Blaha, 1995). Los bovinos son resistentes al M. avium y M. tuberculosis, pero tienen im- portancia en los programas de control, dado que los bovinos se sensibilizan inespecíficamente a la tuberculina y ocasiona problemas en el diagnóstico (Acha y Szyfres, 2003).

El diagnóstico de la tuberculosis bovina se basa en la prueba intradérmica de la tuberculina (PPD) y permite descubrir el 9698\% de los animales infectados (Beer, 1981; Blaha, 1995). Esta prueba ha sido la base de todos los esquemas de erradicación de la tuberculosis que incluyen la detección y el ulterior sacrificio de los animales infectados (Tizard, 2002). Existen varias maneras de realizar la prueba de la tuberculina en el ganado bovino, siendo la más simple la prueba intradérmica única (PIU). En esta prueba se inyecta el PPD en el pliegue caudal y se examina el sitio de inyección 72 horas más tarde. Una reacción positiva consiste en una tumefacción difusa, caliente e indurada en el sitio de la inyección, la cual es fácil de identificar (Tizard, 2002).

Una prueba de inmunoabsorbancia ligada a enzimas (ELISA) que examine los anticuerpos para definir los antígenos de $M$. bovis antes y después de la prueba dérmica puede ser muy útil para detectar animales con reacción positiva inespecífica (Radostits et al., 2002). El ELISA podría ser de utilidad, como prueba complementaria de la intradérmica, para detectar en un rebaño los animales tuberculosos anérgicos que presentan un riesgo para el resto del rebaño (Acha y Szyfres, 2003; Estrada-Chávez et al., 2001; Ritacco et al., 1991). Sin embargo, hasta que no haya sido probada ampliamente en la práctica, el método de elección para la detección de portadores de la tuberculosis con fines prácticos sigue siendo la prueba intradérmica única (Radostits et al., 2002).

La mayoría de explotaciones ganaderas en el Perú no siguen un programa de control y erradicación de la enfermedad, de allí la necesidad de supervisar el aspecto sanitario del hato en previsión de detectar la presencia de enfermedades infectocontagiosas de importancia en salud pública. Frente a esta 
problemática, el Servicio Nacional de Sanidad Agraria (SENASA) ha implementado el Programa de Control y Erradicación de Tuberculosis Bovina en las principales cuencas lecheras del país, teniendo como objetivo la declaración progresiva de áreas libres de esta enfermedad (SENASA, 2000b). En base a esto el objetivo del presente trabajo fue determinar la presencia de tuberculosis bovina en la provincia de Canta empleando la prueba intradérmica única (PIU) en el pliegue caudal.

\section{Materiales y Métodos}

\section{Lugar de estudio}

El estudio se realizó en la provincia de Canta, departamento de Lima, el cual comprende los distritos de Canta, Huamantanga, Huaros, Lachaqui, San Buenaventura, Arahuay y Santa Rosa de Quives. La zona presenta altitudes entre los 550 y 4,450 msnm, con una temperatura promedio anual entre los 5 y $18{ }^{\circ} \mathrm{C}$ y una precipitación pluvial anual de $465 \mathrm{~mm}$. Tiene una topografía accidentada en la parte alta y plana en la parte baja y comprende los pisos ecológicos de yunga, quechua, suni y puna (Vega del Pozo, 2003).

\section{Animales}

El trabajo se realizó en 503 bovinos criollos de ambos sexos pertenecientes a los siete distritos de la provincia de Canta. La edad mínima de los animales fue de cuatro semanas (SENASA, 2000a). El tipo de ganado bovino predominante fue el criollo cruzado con Holstein y Brown Swiss.

\section{Prueba de tuberculina}

Se aplicó una dosis de $0.1 \mathrm{ml}$ de PPD bovino (5,000 UI ó $1 \mathrm{mg} / \mathrm{ml})$ en el pliegue de la cola, según el Reglamento del Programa de Control y Erradicación, en su capítulo V, artículo 15 (SENASA, 2000a).

La correcta inoculación se constató mediante la observación y palpación de una pequeña pápula en el punto de inoculación del PPD (Errico, 1989). La lectura se hizo después de 72 horas de la inoculación detectando una posible inflamación (tumefacción difusa e indurada). Todo cambio palpable o visible en la zona de inoculación fue considerada como reacción positiva (OIE, 1996).

\section{Análisis de datos}

Los datos obtenidos y considerando los valores de sensibilidad y especificidad del PPD fueron sometidos a la técnica de evaluación de riesgo por simulación Monte Carlo (programa @Risk) con un nivel de confianza del $99 \%$.

\section{Resultados}

Se encontró 11 animales reactores a la prueba de tuberculina (11/503), representando el 2.2\% (Cuadro 1). La técnica de evaluación de riesgo por simulación Monte Carlo (programa@Risk) indicó que la probabilidad de encontrar la infección en el ganado bovino en la provincia de Canta es mayor al $1 \%$ y menor al $3.2 \%$.

\section{Discusión}

La prevalencia corregida $(<3.2 \%)$ es mayor a los valores reportados por estudios realizados por el SENASA para el control y erradicación de tuberculosis bovina en otras partes del país, en los cuales se detectó prevalencias relativamente bajas. Así por ejemplo, 0.17\% en Junín (SENASA, 1999), $0.65 \%$ en Cajamarca, $0.21 \%$ en Puno, $0.075 \%$ en Arequipa, en tanto que en Cuzco no se detectaron animales reactores. En todos estos lugares la tuberculinización fue realizada en el pliegue caudal (SENASA, 2000b). La prevalencia de la tuberculosis bovina fue muy variable entre distritos, zonas y regiones, tanto en este trabajo como en otros (Samara et al., 1996). 
Cuadro 1. Frecuencia de bovinos reactores a la prueba de tuberculina en distritos de la provincia de Canta, Lima

\begin{tabular}{lccc}
\hline & Animales muestreados & \multicolumn{2}{c}{ Animales reactores } \\
\cline { 3 - 4 } Distrito & $(\mathrm{n})$ & $\mathrm{n}$ & $\%$ \\
\hline Canta & 107 & 1 & 0.2 \\
Huamantanga & 126 & 1 & 0.2 \\
Huaros & 84 & 1 & 0.2 \\
Lachaqui & 73 & - & 0 \\
San Buenaventura & 41 & - & 0 \\
Arahuay & 32 & 1 & 0.2 \\
Santa Rosa de Quives & 40 & 7 & 1.4 \\
\hline Total & 503 & 11 & 2.2 \\
\hline
\end{tabular}

La prevalencia encontrada en el estudio pudo ser influenciada por el tipo de explotación, la introducción de animales en los hatos, el manejo y el medio ambiente. El tipo de explotación que se realiza en Canta es semiextensiva. En general, en distritos como Canta, Huaros, San Buenaventura y Arahuay, distritos que tienen una mejor disponibilidad de pastos, los animales permanecen una semana en promedio en cada potrero. En los distritos de Lachaqui y Huamantanga, los animales se mantienen en los potreros por un tiempo más prolongado. Estos distritos no poseen gran disponibilidad de pastos y en el tiempo en que se realizó el estudio estos lugares se encontraban en sequía.

El distrito de Santa Rosa de Quives tuvo la mayor frecuencia de animales reactores a la tuberculina. Aquí los animales permanecen la mayor parte del tiempo en corrales, aunque salen a pastar en la madrugada hasta la hora del ordeño y luego en la tarde hasta antes del anochecer.

Al no haber coexistencia continua entre los animales, como ocurre en los establos de explotación lechera intensiva, en donde además de la coexistencia tiene que ver el uso común de comederos y bebederos, se encontró una prevalencia bastante baja. La excepción fue en Santa Rosa de Quives, cu- yos animales tienen un manejo cercano al tipo de explotación intensiva.

Es importante mencionar el riesgo del tránsito de animales sin un debido control sanitario. Los ganaderos de la provincia de Canta obtienen el ganado principalmente del norte de la provincia de Lima, región que tiene una alta prevalencia de tuberculosis bovina (A. Delgado, Comunicación personal). En este sentido, el distrito de Santa Rosa de Quives por encontrarse limítrofe con la región norte de la provincia de Lima, se encuentra con un mayor riesgo para la introducción de animales infectados de tuberculosis bovina.

Zonas altas como el distrito de Lachaqui $(3,700 \mathrm{msnm})$ tiene un clima frío que impide la supervivencia del $M$. bovis, mientras que el distrito de Santa Rosa de Quives presenta un clima cálido (Vega del Pozo, 2003), favorable para la difusión de la tuberculosis bovina.

Finalmente es necesario recordar que los animales reactores positivos a la prueba de tuberculina pueden haber sido falsos positivos por una reacción inespecífica a infecciones anteriores por $M$. bovis, M. avium, M. tuberculosis, M. paratuberculosis o Nocardia farcinicus (Blaha, 1995; Radostits et al., 2002). Los reactores positivos pueden 
haber sido infectados también por sus propietarios con $M$. tuberculosis o por los otros agentes mencionados anteriormente que se encuentran en el medio ambiente.

En el presente trabajo se realizó la prueba de intradermorreacción única (PIU) en el pliegue caudal (prueba obligatoria en el Perú y en muchos países donde se implanta un programa de control y erradicación); la cual presenta la ventaja de ser una prueba simple y es realizada en lugares donde se desconoce el estado zoosanitario de esta enfermedad (Errico, 1989; Ortiz, 1986; Villamil, 1990), aunque tiene limitaciones por poseer una sensibilidad promedio del 80\% (OPS/OMS, 1992, Francis et al., 1978). Sin embargo, sigue siendo considerada la prueba de campo ideal a usarse en casos como el presente estudio.

\section{Conclusión}

La tuberculosis bovina tiene una prevalencia mayor al $1 \%$ y menor al $3.2 \%$ en el ganado bovino de la provincia de Canta, Lima.

\section{Literatura Citada}

1. Acha, P. N.; B. Szyfres. 2003. Zoonosis y enfermedades transmisibles al hombre y animales. Vol. I. $3^{\text {a }}$ ed. p 266-280. Organización Panamericana de la Salud. Washington DC.

2. Beer, J. 1981. Enfermedades infecciosas de los animales domésticos. Tomo II. p 229-249. Ed. Acribia. España.

3. Blaha, T. 1995. Epidemiología especial veterinaria. Ed. Acribia. p. 164-172 España.

4. Castagnino, D. 1968. Resultados del muestreo de tuberculosis bovina en el Perú. Tercer Boletín Extraordinario. p 158-162. IVITA- Facultad de Medicina Veterinaria, Univ. Nacional Mayor de San Marcos. Lima.
5. Errico, F. 1989. Guía técnica de métodos y criterios de interpretación de la prueba tuberculínica en bovinos. En: Programas de control y erradicación de la tuberculosis, brucelosis bovina y fiebre aftosa. IICA. p 1-11. Arequipa, Perú.

6. Estrada-Chávez, C.; R. Mancilla; $C$. Arriaga; R. Pérez; F. Díaz. 2001.

Determinación de anticuerpos anti-PPD en hatos lecheros con distintas prevalencias de tuberculosis bovina en méxico. Vet. Méx. 32: 207-210.

7. Francis, J.; R.S. Séller; I.W. Wilkie; D.O'Boyle; M.J. Lumsden; A.J. Frost. 1978. The sensibility and specificity of various tuberculin test using bovine PPD and other tuberculins. Vet. Rec. 103: 420-425.

8. Langeneger, C.H.; J. Oliveira. 1981. Tratamento da tuberculose bovina com isoniazida. Pesq. Vet. Bras. 1: 1-6.

9. OIE. 1996. Tuberculosis bovina. Manual OIE. p 267-275. París.

10. Ortiz, R. 1986. Cuba, Erradicación de la tuberculosis bovina. Revista Mundial de Zootecnia 59: 34-37.

11. OPS/OMS. 1992. Plan de acción para la erradicación de la tuberculosis en las Américas. Fase I. p 5-40. Washington D.C. USA.

12. O'Reilly, L.; C. Daborn. 1995. The epidemiology of Mycobacterium bovis infections in animals and man: a review. Tuberc. Lung. Dis. 76 (supl): 1-46.

13. Radostits, O.; O. Blood; C. Gay; K. Hinchcliff. 2002. Medicina veterinaria. Tratado de las enfermedades del ganado bovino, ovino, porcino, caprino y equino. $9^{a}$ ed. p 1076-1085. Ed. Mc GrawHill Interamericana. España.

14. Ritacco, V.; B. López; I. N. De Kantor; L. Barrera; F. Errico; A. Nader. 1991. Diagnostic tests of the bovine tuberculosis. Res. Vet. Sci. 50: 365-367.

15. Samara, S.; M. Buzinaro; M. Oliveira. 1996. Diagnóstico da situaçao sanitária do gado leiteiro em Pitangueiras-SP: I Tuberculose. Rev. Med. Veterinaria e Zootecnia 12: 132-135. 
16. SENASA. 2000a. Reglamento para el control y erradicación de la tuberculosis bovina. D.S. $N^{\circ}$ 31-2000-AG. Disponible en: http: //www: portalagrario.gob.pe/ legales/18944a.htm

17. SENASA. 2000b. Control y erradicación de tuberculosis y brucelosis bovina. Manual de procedimientos. Dirección General de Sanidad/Dirección de Programas Zoosanitarios. p 1-9. Lima, Perú.

18. SENASA. 1999. Programa de brucella y tuberculosis bovina en el departamento de Lima. Perú. p 1-12.

19. Sommerfelt, I. 1989. Epidemiología de la tuberculosis bovina. En: Programas de control de erradicación de la tuberculo- sis, brucelosis bovina y fiebre aftosa. $\mathrm{p}$ 1-6. IICA. Arequipa, Perú. OPS.

20. Tizard, I. 2002. Inmunología veterinaria. $6^{a}$ ed. p 371-379. Ed. Mc Graw-Hill Interamericana. España.

21. Toledo, P.; M. Santillán; F. Milián; I. Ramírez. 1999. Aislamiento e identificación de Mycobacterium bovis a partir de muestras de expectoración de pacientes humanos con problemas respiratorios. Vet. Méx. 30: 227-229.

22. Vegas del Pozo, J. 2003. A propósito del desarrollo del capitalismo. Rev. Antropol. 83-105.

23. Villamil, L. 1990. Notas sobre la epidemiología de la tuberculosis con énfasis en bovinos. El Cebú 254: 42-48. 